\title{
Pulsation and Evolution. An observer's viewpoint
}

\author{
C. Waelkens \\ Instituut voor Sterrenkunde, Katholieke Universiteit Leuven, \\ Celestijnenlaan 200 B, B-3001 Heverlee, Belgium
}

\begin{abstract}
We review observational evidence on the interaction between stellar pulsation and evolution. We discuss to what extent observations of pulsating stars with variable amplitudes and pulsation periods have implications on our understanding of stellar structure and evolution. The probable link between mass loss and pulsation in AGB stars and in hot luminous stars appears to be the strongest way in which pulsations affect evolution. We point out the possibility that forced oscillations in the components of binaries may have important consequences on evolution, that could offer an explanation for some classes of peculiar evolved objects.
\end{abstract}

\section{Introduction}

Evolution is the central paradigm that characterizes most astrophysical research, if not natural sciences in general. Confronting stellar pulsations with stellar evolution theory is therefore a key issue, both from the theoretical and observational points of view. Because, however, evolution and pulsation timescales differ so widely, direct confrontation of both is not an easy matter, and most evidence is of an indirect nature.

In this review, we will first address the question how evolution affects pulsation. Obvious aspects are observations of stars entering or leaving the various instability strips in the HR diagram, and the variations of pulsation periods. While the available evidence is rather consonant with stellar evolution theory, it is a striking fact that it probably tells us more about stellar structure than about evolution. Since observed stellar pulsations most often do not significantly affect the deep interior of stars, it is rare that pulsation affects evolution. An important issue is, however, pulsation-induced mass loss, for which evidence exists in hot massive stars as well as in evolved low-mass stars.

The last part of this review is devoted to the possible role resonant forced oscillations may play in the evolution of components of binaries. There is no hard direct evidence for such phenomena, but some indirect arguments can be put forward that they may be significant.

\section{Onset and decay of pulsation}

The very existence of well delimited instability strips in the HR diagram evidently is the best proof that evolution affects pulsation. A useful ch sck is the statistics of variable stars versus constant stars. The population of the Cepheid 
strips is now well understood in terms of the excursions massive supergiants make from and back to the red supergiant stage. As far as Miras are concerned, the fact that these stars outnumber the planetary nebulae only by a factor 3 indicates that the duration of the Mira stage is rather short, i.e., of the order of $610^{4}$ years only (Wood 1990), which may be seen as an indication of the effect of pulsation on evolution (see below).

The observational record of stars entering an instability strip and so starting pulsation, is very poor. Of course, no observer will commit himself to the monitoring of a constant star, waiting for its incipient pulsation. In these proceedings, Barlai presents evidence for an RR Lyrae star in M15 that may be in the process of starting its pulsations. The prospects of detecting the onset of Mira-type pulsations are much better now: comparing numbers and timescales, one finds that this should happen in the LMC once in 40 years, a fact which should prompt the EROS and MACHO teams to preserve their database for future generations.

Some well documented cases exist of stars with declining pulsation amplitudes, eventually stopping pulsating completely, e.g., the Cepheids Y Oph (Fernie 1990) and Polaris (Fernie et al. 1993), and the $\beta$ Cephei star $\alpha$ Virginis. All cases, however, concern objects that are rather centrally situated in the respective instability strips, and it seems that the evanescent nature of the pulsations is an effect of changing internal structure rather than of evolution. A better case for a star leaving the instability strip may be RU Cam (Teays 1990).

A remarkable case is the peculiar Cepheid HR 7308, the amplitude of which undergoes a modulation on a timescale of some 1000 days. A new explanation for this object has been put forward by Van Hoolst \& Waelkens (1995), who interpret its behaviour as a resonant interaction between the second radial overtone and a non-radial mode with approximately the same period. This interpretation involves a 1-1 resonance in which energy is cyclically exchanged between a non-radial mode, which is pulsationally stable by itself, and an unstable radial mode. Such a 1-1 resonance can only happen for the second and higher overtones; it would be of interest to find out whether similar behaviour is present in some of the second overtone pulsators discovered by the MACHO project in the Magellanic Clouds (Welch, this conference).

\section{Period variations}

The change of the internal structure of a star during its crossing of an instability strip must affect its period. Such period changes have been searched for and found in several classes of pulsating variables. However, in most cases, it appears that the period variations caused by internal structural adjustments of another nature dominate the small effects of evolution, so that the diagnostic value of these results for stellar evolution purposes is somewhat deceiving.

Not unexpectedly, the clearest results have been found for Cepheids, for which a long record exists and which evolve on a relatively short timescale. A general result which emerges, is that the period increases and decreases allow one to locate Cepheids on the various paths through the instability strip, the statistics of which are in good qualitative agreement with stellar evolution (Fernie 1984). For RR Lyrae stars, random changes due to internal adjustments 
often dominate (Sweigart \& Renzini 1979; Stothers 1980). On the other hand, Lee (1991) found a significant evolutionary increase in the periods of RR Lyrae stars in Oosterhoff II clusters (but see also Barlai, this conference).

For Mira's period changes are dominated by random effects (Percy et al. 1990), a view consistent with Whitelock's (1990) suggestion that hardly any evolutionary period change occurs for these stars. This suggests that the period increase due to a luminosity increase is compensated by the decrease due to mass loss, and fits well into a picture of a short-lived Mira phase. For RV Tauri stars, which according to Jura (1986) are rapidly evolving post-AGB stars, the situation is more confusing. While for $\mathrm{R}$ Scuti a period decrease consistent with rapid evolution is observed (Percy et al. 1991), the agreement is less good for U Mon, and the period of AC Her even displays an oscillatory behavior (Zsoldos 1988). An interesting result is that the absence of rapid period variations in 89 Her pleads against rapid post-AGB evolution for this star (Fernie \& Sasselov 1989), in good agreement with the fact that this object has a Flamsteed number!

For main-sequence pulsators, the expected evolutionary changes are low, and observations show that other effects dominate. In some $\beta$ Cephei and some $\delta$ Scuti stars period increases and decreases are observed simultaneously for different modes. There is no disagreement with evolution theory, but the diagnostic value of period variations for stellar evolution is low. A notable exception may be BW Vul, for which Sterken \& Jerzykiewicz (1990) claimed that the rapid period increase points to a post-core-hydrogen-burning evolution, which would put the exceptionally large pulsation amplitude of this star into a new perspective. Another most interesting result is the systematic presence of period decreases for evolved $\delta$ Scuti stars (Breger, this conference).

Extensive and detailed observations exist on the period variations of pulsating degenerate objects, where again structural and evolutionary effects interfere. The interpretation of the observations of white-dwarf pulsations is treated elsewhere in this volume by Kawaler.

We are then tempted to conclude that the observations of period changes in pulsating stars are useful though most often not conclusive for stellar evolution purposes. This conclusion should not discourage us to continue such observations, because of the wealth of other information they contain. Pulsation period variations may be the best diagnostic for probing the random structural changes inside stars, and have often led to the very important discovery of orbital motion of pulsating stars.

\section{Pulsations and mass loss}

Stars at the luminous tip of the AGB all are large-amplitude pulsating variables and all undergo severe mass loss. Both the large pulsation amplitudes and the mass loss are linked to the low gravity of these stars. A crucial question is whether a more direct link exists between pulsation and mass loss, i.e., is the mass loss induced by the pulsations? If not, the coexistence between mass loss and pulsation on the tip of the AGB would just be a coincidence, and the mass loss may be triggered by other mechanisms, such as thermal pulses or hydrogen recombination instabilities (Wagenhuber \& Weiss 1994). 
According to theoretical computations (Bowen 1988; Bowen \& Willson 1991; Feuchtinger et al. 1993) the shocks associated with Mira pulsations increase the scale height of the atmosphere of an AGB star so that the density in its external, low-temperature, layers is sufficiently high for dust to form; radiation pressure then accelerates the dust which drags the gas with it.

An observational difficulty for the Bowen scenario is the fact that an extended post-shock region would produce molecular emission, which is not observed (Bessell et al. 1989). Second, direct observations of detached circumstellar shells and accurate modeling of energy distributions suggest that the mass loss is not a continuous process. Episodic mass loss is then probably related to thermal pulse phases. This is not necessarily an argument against the pulsation-inducedmass-loss hypothesis, since also pulsation amplitudes vary with the changing interpulse luminosity. On the other hand, various observations suggest a causal link between pulsation and mass loss. The fact that a large fraction of dustenshrouded AGB stars are Miras suggests more than just a coincidence. Furthermore, a clear correlation exists between pulsation amplitude and mass loss rates (Whitelock 1990). In my opinion, the strongest argument is the short duration of the Mira phase, as it is computed from statistics in our Galaxy and the LMC: once a star becomes a Mira, its days on the AGB are numbered.

Some relation between pulsation and mass loss has also since long been suspected for hot luminous stars. We do not have to enter the discussion of pulsations of $\mathrm{Be}$ stars, since in these objects the mass loss hardly affects the evolution. More important for stellar evolution is the long-standing mystery of the origin of the very high mass loss rates of Wolf-Rayet stars. Maeder (1985) proposed that WR stars are vibrationally unstable. From the finding by Glatzel et al. (1993) that stars on the helium main sequence are violently unstable, Langer et al. (1994) elaborated on the effects of these pulsations on mass loss. The pulsations would lift the mass loss beyond the sonic point, where the radiation-driven wind would take over, in a way somewhat reminiscent of what is seen in AGB stars.

From the direct observational point of view, the situation with WR pulsations is somewhat frustrating. Blecha et al. (1993) announced the first direct observation of pulsations in WR40, but these observations could never be reproduced, not by themselves, nor by any other group. An observational caveat clearly is that the photospheres of these stars (if they can be defined) are hardly ever seen because of the dense wind. By all means, if WR pulsations have been observed once, they should be confirmed soon rather than late.

Violent pulsational instabilities are now also found for evolved hydrogen burning very massive and luminous stars (Glatzel \& Kiriakidis 1993a, 1993b). It appears that the incorporation of the new opacities to stellar models implies that pulsational instabilities are inescapable in the upper part of the HR diagram, where the Cepheid and $\beta$ Cephei instability strips converge in the region where luminous blue variables are observed (Dziembowski 1992). According to Langer et al. (1994), the regions in the HR diagram of largest pulsational instability and of highest mass loss rates are coincident. The present situation thus holds much promise that the long-standing problem of the high mass loss rates of very massive stars may be reaching a solution, in which pulsations would play a major role. 


\section{Forced stellar oscillations}

Among late-type giants and supergiants, a diverse zoo exists of objects the peculiarities of which can only be explained by mass transfer in a binary system. The Barium stars consist of an s-process enhanced star orbiting a white-dwarf. Since these objects have survived as fairly wide systems, it is excluded that the star that presently is a white dwarf has ever filled its Roche lobe, since then fast mass transfer and spiral-in would have occurred. However, some systems presently are so close that no Mira would fit into the Roche lobe of the white dwarf.

Tout \& Eggleton (1988) have proposed that such close systems can be explained if one assumes that heavy mass transfer, induced by the tidal interactions, has occurred well before the AGB star filled its Roche lobe. This mass loss would then have impeded the star to grow to the size of typical single AGB stars, so that the decrease of the size of the system due to mass transfer never led to catastrophic mass transfer, and relatively wide systems with a white-dwarf component could survive. There is no clear understanding, however, of the way tidal interactions in well detached systems can increase mass loss rates by two orders of magnitude, as proposed by Tout \& Eggleton.

A similar problem is encountered in some objects assumed to be post-AGB stars, such as HR 4049 and HD 44179, the central star of the Red Rectangle nebula. The latter object is particularly interesting, because it displays a remarkable bipolar nebula. That previous AGB evolution is involved for these objects is attested by the carbon richness of their circumstellar environment. Both objects occur in binaries with orbital periods that are much too short (434 and 298 days) to have accommodated a terminal AGB star of normal size (Van Winckel et al. 1995). Also the RV Tauri star 89 Herculis occurs in a binary, with an orbital period of 288 days (Waters et al. 1993).

Since observational evidence is accumulating that the high mass loss rates of AGB stars are linked to pulsations, it is worthwhile investigating this hypothesis for these peculiar binaries. In no case can a Mira fit into the system without destroying it. However, we propose that stellar oscillations may have occurred in these objects in an earlier phase, as forced oscillations by the companion. Indeed, since an AGB star undergoes substantial variations of size in a relatively short period, it appears hardly escapable that at some point a resonance develops between a free oscillation mode of the AGB star and the orbital motion. If during this resonance orbital energy is transfered to the oscillation mode, it could make the latter grow to macroscopic amplitudes, and cause pulsation-induced mass loss.

Ruymaekers \& Smeyers (1994) have developed a theoretical framework for the passage through resonance of a dynamic tide in a rapidly evolving star. They find that such a passage indeed does affect the radial component of the tidal displacement. It would certainly be interesting to apply this theory to highorder g-modes in evolving AGB stars. If a positive answer is found, it would provide an independent way of probing the relation between pulsation and mass loss.

An interesting aspect in both HR 4049 and HD 44179 is that the present orbits are fairly eccentric. At first sight, a large eccentricity complicates the picture still further, in the sense that mass transfer and tidal interactions usually 
tend to circularize stellar orbits. However, it is not yet clear how the forced oscillations affect the eccentricity of the orbit. Moreover, observations by Matthieu (1992) and theoretical developments by Artymovicz et al. (1991) have shown that binary stars probably get their eccentricity before the main sequence, from tidal interactions with the circumsystem disk. Tidal interactions with the postAGB circumsystem disk which is presently observed around both HR 4049 and HD 44179 may then also have been responsible for the large present eccentricity of these systems.

\section{References}

Artymovicz, P., Clarke, C., Lubow, S.H., \& Pringle, J.E. 1991, ApJ, 370, L35

Bessell, M.S., Brett, J.M., Scholz, M., \& Wood, P.B. 1989, A\&A, 213, 209

Blecha, A., Schaller, G., \& Maeder, A. 1993, Nature 360, 320

Bowen, G.H. 1988, ApJ, 329, 299

Bowen, G.H., \& Willson, L.A. 1991, ApJ, 375, L53

Dziembowski, W.A. 1992, in: Pulsation, Rotation and Mass Loss in Early-Type Stars, Proc. IAU Symposium No. 162, eds. L.A. Balona, H.F. Henrichs \& J.M. Le Contel, p.55

Fernie, J.D. 1990, PASP, 102, 905

Fernie, J.D. 1994, in: Observational Tests of the Stellar Evolution Theory, Proc. IAU Symposium No. 105, eds. A. Maeder \& A. Renzini, Reidel, Dordrecht, p. 441

Fernie, J.D., Kamper, K.W., \& Seager, S. 1993, ApJ, 416, 820

Fernie, J.D., \& Sasselov, D.D. 1989, PASP, 101, 513

Feuchtinger, M.U., Dorfi, E.A., \& Höfner, S. 1993, A\&A, 273, 513

Glatzel, W., \& Kiriakidis, M. 1993a, MNRAS, 262, 85

Glatzel, W., \& Kiriakidis, M. 1993b, MNRAS, 263, 375

Glatzel, W., Kiriakidis, M., \& Fricke, K.J. 1993, MNRAS, 262, L7

Jura, M. 1986, ApJ, 309, 732

Langer, N., Hamann, W.R., Lennon, M., Najarro, F., Pauldrach, A.W.A., \& Puls, J. 1994, A\&A, 290, 819

Lee, Y.W. 1991, ApJ, 367, 524

Maeder, A. 1985, A\&A, 147, 300

Matthieu, R.D. 1992, in: Binaries as Tracers of Stellar Formation, eds. A. Duquennoy \& M. Mayor, Cambridge University Press, p.155

Percy, J.R., Colivas, T., Sloan, W.B., \& Mattei, J.A. 1990, in: Confrontation between Stellar Pulsation and Evolution, eds. C. Cacciari \& G. Clementini, ASP Conf. Series, p.446

Percy, J.R., Sasselov, D.D., Alfred, A., \& Scott, G. 1991, ApJ, 375, 691

Ruymaekers, E., \& Smeyers, P. 1994, A\&AS, 104, 401

Sterken, C., \& Jerzykiewicz, M. 1990, in: Confrontation between Stellar Pulsation and Evolution, eds. C. Cacciari \& G. Clementini, ASP Conf. Series, p. 236 
Stothers, R. 1980, PASP, 92, 475

Sweigart, A.V., \& Renzini, A. 1979, A\&A, 71, 66

Teays, T.J. 1990, in: Confrontation between Stellar Pulsation and Evolution, eds. C. Cacciari \& G. Clementini, ASP Conf. Series, p.128

Tout, C.A., \& Eggleton, P.P. 1988, MNRAS, 231, 823

Van Hoolst, T., \& Waelkens, C. 1995, A\&A, 295, 361

Van Winckel, H., Waelkens, C., \& Waters, L.B.F.M. 1995, A\&A, 293, L25

Wagenhuber, J., \& Weiss, A. 1994, A\&A, 286, 121

Waters, L.B.F.M., Waelkens, C., Mayor, M., \& Trams, N.R. 1993, A\&A, 269, 242

Wood, P.R. 1990, in: Confrontation between Stellar Pulsation and Evolution, eds. C. Cacciari \& G. Clementini, ASP Conf. Series, p.355

Whitelock, P.A. 1990, in: Confrontation between Stellar Pulsation and Evolution, eds. C. Cacciari \& G. Clementini, ASP Conf. Series, p.365

Zsoldos, E. 1988, Inf. Bull. Variable Stars 3192

\section{Discussion}

Habing: Estimating the number of PNe versus the number of Miras is dangerous: in our Galaxy the number of PNe is uncertain by a factor of 10 .

Waelkens: I believe that the statistics of Miras are more reliable than that. So, if there are much more planetary nebulae, that would shorten Mira lifetimes still more. On the other hand, the census in the LMC should be fairly complete, and it is in agreement with the present statistics for our Galaxy.

Whitelock: I was interested in your comments about binary and pulsation interaction as a cause for mass ejection in AGB/post-AGB stars. It has worried me for some while that what we refer to as post-AGB phenomena may be associated with binary interaction. Do you know of any 'post-AGB' stars that are clearly solitary?

Waelkens: Yes. It seems that single stars have IR excesses only at IRAS wavelengths, while binaries also have near-IR excesses. Good candidate single postAGB stars are HD 161796, HD 187885 and a few others

Feast: One would not expect a large amplitude Mira to start up suddenly. The evidence (e.g., globular clusters) shows that Miras are part of a wider instability strip which includes semi-regular (lower amplitude) variables as a prior evolutionary phase.

Waelkens: I cannot predict how Mira pulsations would start. My point is that the extensive coverage of the LMC by the MACHO and EROS projects may show us how it happens, once every 50 years or so.

Mattei: In the AAVSO Observing Program there are about 50 semi-regular variables that had large amplitudes in the past and presumably have much smaller amplitudes now. Bob Cadmus from Grinnell College is monitoring them photoelectrically. These stars deserve attention for they may evolve into something 
else other than being long period variables. Also I want to report that the 75year AAVSO data on maxima and minima dates of long period variables, used extensively to search for period changes, will soon be extended to 90 years with the addition of data from 19755 to 1990.

Dziembowski: What could be the non-evolutionary structural changes causing period changes?

Waelkens: We can only guess. Earlier this morning we were told that mixing processes should be important. Such processes may also occur in discontinuous events. Remember that mass loss was first considered to be a continuous process, but now there is plenty of evidence that mass loss occurs in bursts.

Burki: Concerning HR 7308, I was in favour of a star evolving at the border of the instability strip, because the number of cycles between successive extrema of amplitude is in good agreement with the theoretical predictions by J. and A. Cox. Of course, in this case, we need an interaction between evolution and pulsation. In your proposed explanation, have I correctly understood that there is not an addition of two modes and that they cannot be observationally, separately, detected?

Waelkens: The basic idea is that there is one unstable radial mode which transfers energy to another, normally stable, non-radial mode with about the same period. As the amplitude of the radial mode declines and that of the non-radial one grows, damping of the non-radial mode starts and the radial one takes over again. Such a one-to-one resonance can only occur from the second radial overtone on, but then it is not unlikely, since the non-radial mode spectrum is rich in the vicinity of the second radial overtone. You have convincingly shown that HR 7308 is a second overtone pulsator.

Percy: (i) With regard to RV Tauri stars: the apparent period changes may be due to random fluctuations, as with the Mira stars. Endre Zsoldos and I have begun a project to test this hypothesis. (ii) For the Mira stars: because we have data on about 400 stars over more than 75 years, we may be able to average out the random fluctuations and detect the evolutionary changes.

Waelkens: Both projects are very important and underscore the interest of the AAVSO database. If no evolutionary period changes are found for the RV Tauri stars, or a least for some of them, that would have serious implications for our understanding of these stars in terms of stellar evolution. 